MPI-Ph/93-07

TUM-TH-152/93

hep-th/9303017

February 1993

\title{
Threshold Corrections to Gauge Couplings in Orbifold Compactifications*
}

\author{
P. Mayr and S. Stieberger \\ Physik Department \\ Institut für Theoretische Physik \\ Technische Universität München \\ D-8046 Garching, FRG \\ and \\ Max-Planck-Institut für Physik \\ -Werner-Heisenberg-Institut- \\ P.O. Box 401212 \\ D-8000 München, FRG
}

\begin{abstract}
We derive the moduli dependent threshold corrections to gauge couplings in toroidal orbifold compactifications. The underlying six dimensional torus lattice of the heterotic string theory is not assumed - as in previous calculations - to decompose into a direct sum of a four-dimensional and a two-dimensional sublattice, with the latter lying in a plane left fixed by a set of orbifold twists. In this more general case the threshold corrections are no longer automorphic functions of the modular group, but of certain congruence subgroups of the modular group. These groups can also be obtained by studying the massless spectrum; moreover they have larger classes of automorphic functions. As a consequence the threshold corrections cannot be uniquely determined by symmetry considerations and certain boundary conditions at special points in the moduli space, as was claimed in previous publications.
\end{abstract}

* Supported by the Deutsche Forschungsgemeinschaft and the EC under contract SC1-CT92-0789. 


\section{Introduction}

String theory is the only known theory which consistently unifies all interactions. To make contact with the observable world one constructs the field-theoretical low-energy limit of a given ten-dimensional string theory. To get a four-dimensional effective $\mathrm{N}=1$ supergravity theory one compactifies six of the ten dimensions on an internal Calabi-Yau manifold [1] or a toroidal orbifold [2]. In addition one integrates out all the massive string modes corresponding to excited string states as well as states with momentum or winding numbers in the internal dimensions. The resulting theory then describes the physics of the massless string excitations at low energies in field-theoretical terms. One question of current interest are threshold corrections to the gauge couplings due to the infinite tower of massive string modes. Since they provide the boundary conditions for the running gauge couplings they are the foundation of any discussion about gauge coupling unification.

One property of string theory is that there are infinitely many vacua labeled by the vacuum expectation value of certain scalar fields: the dilaton field and the moduli fields. The latter parametrize the shape and the size of the compactification manifold. They enter the lowenergy theory as massless scalar fields with flat effective potentials neglecting non-perturbative effects. The moduli space of string vacua is invariant under certain discrete reparametrizations, e.g. target space duality. It is clear that the limiting effective supergravity theory should reflect these stringy symmetries. In particular it has to be anomaly free with respect to the discrete reparametrizations. Since the supergravity Lagrangian for the massless fields produces anomalous triangle graphs [3, 1,0 , 5], anomaly freedom has to be restored by adding appropriate counterterms. Indeed the full string theory generates two kinds of such counterterms: an universal gauge-group independent Green-Schwarz term and the threshold corrections arising from the contribution of heavy string modes in the loops [3].

The moduli dependence of the effective action can be determined by calculating the appropriate string scattering amplitudes. In particular this point has been discussed for the Kähler potentials [6], thresholds corrections to the gauge couplings [7] as well as gravitional and Yukawa couplings [8, 9] in a general framework. More concrete results can be obtained for orbifold compactifications: This issue was discussed for the Kähler potentials [10], Yukawa couplings [11] and threshold corrections to the gauge couplings due to the influence of the massive string modes [12, 9]. Threshold corrections to the gauge couplings play a crucial rôle for anomaly cancellation in the effective Lagrangian [3] as well as for string unification [13, 14]. The modular invariant threshold corrections to the gauge couplings evaluated in ref. [12] can be considered to split into two parts: The first is interpreted to be that of the massless states and is the analogue of the field theoretical triangle graph involving two gauge fields and one auxiliary connection corresponding to either the Kähler connection or the sigma model connection [3, 4, 5]. This term transforms anomalous under the discrete reparametrizations on the moduli space. The other part is holomorphic and describes a modification of the effective Lagrangian due to the contribution of super-heavy string modes in the loops. From the point of field theory this term shows up as a counterterm repairing the duality anomaly of the first term. On the other hand the threshold corrections provide the boundary conditions for the renormalization group equations of the running gauge couplings and from the field-theoretical point of view thus determine the unification mass $M_{\mathrm{X}}$. They may shift $M_{\mathrm{X}}$ to the string scale $M_{\text {string }}$ or even to higher values [13, 14]. Finally the threshold corrections to the gauge couplings may also be important for supersymmetry breaking via gaugino condensation 15].

The effective field theory has in general singularities because one has also integrated over massive states which become massless at special points in the moduli space. The large radius behaviour $\operatorname{Im} T \rightarrow \infty$ of the moduli dependence of the one-loop corrections to the gauge couplings was first discussed in ref. [16]. In this limit an infinite number of Kaluza-Klein states 
becomes massless and causes a singularity in the threshold corrections linear in $\operatorname{Im} T$. On the other hand the winding states become massless at the duality transformed point $\operatorname{Im} T=0$. There can be also special points in the moduli space where additional charged fields become massless and give rise to logarithmic poles in the threshold function. The most general function which reproduces this singular behaviour and is in addition target-space modular invariant is given by the expression [17]:

$$
\triangle(T, \bar{T})=-b \ln \left[\operatorname{Im} T|\eta(T)|^{4}\right]+\frac{b}{3} \ln |H(T)|^{2},
$$

where $\eta$ is the Dedekind eta-function and $b$ is related to the one-loop $\beta$-function in a way to be specified later. $H(T)$ is a modular invariant function which has no zeros and poles at $\operatorname{Im} T \rightarrow \infty$ and determines the functional behaviour at the critical points.

In refs. [18, 19] it was shown that the functional dependence of the threshold corrections to the gauge couplings is the same as the one of the topological free energy of that two-dimensional subspace of the full torus lattice, which is left fixed by the orbifold twist. This topological invariant is believed to be invariant under the modular group $\Gamma \equiv S L(2, \mathbb{Z})$ and thus should be an automorphic function of $\Gamma$.

An explicit expression for the moduli dependent threshold correction to the gauge couplings in the case of orbifold compactifications was derived in [12]. Here the sum over all massive string modes was evaluated. It was shown there that only orbifold sectors with one unrotated plane contribute to this function. Since these sectors posses two space-time supersymmetries they are called $\mathrm{N}=2$ sectors. In ref. [12, 9] only the special case was considered, where the six-dimensional lattice splits into a direct sum of a two-dimensional and a four-dimensional lattice $T^{6}=T^{2} \oplus T^{4}$ with the unrotated plane lying in $T^{2}$. The main purpose of this paper is to generalize these calculations to the larger class of orbifold models which do not fulfill this assumption. In fact most of the toroidal orbifolds are of this type [20].

The new feature is the fact that the lattice vectors characterizing momentum and winding states can now have non-vanishing components in all lattice directions. This has to be contrasted with the investigations of refs. [12, 19] where these states had only two non-vanishing components. This novelty has an important consequence: The threshold corrections are no longer invariant under the full modular group $\Gamma$ but only under a certain subgroup of $\Gamma$. Since those subgroups have in general a larger set of automorphic functions, the thresholds will be composed of different terms. As required by consistency, also the topological free energy for these kind of orbifolds shares this property and therefore is no longer invariant under the full modular group.

The organization of this paper is as follows: In section 2 we present the basic formulas necessary to determine the threshold corrections for the general case of orbifolds with no restrictions on the lattice. Then we evaluate the threshold corrections to the gauge couplings for a $Z_{4}, Z_{6}$ and $Z_{8}$ orbifold. We compare the results with the corresponding topological free energies and determine the massless spectrum. As a consequence of the restriction of the threshold symmetry to a subgroup of $\Gamma$ the duality anomaly cancellation terms in the effective action are modified, too. Therefore the boundary conditions for the running gauge couplings and the unification mass change. This will be discussed in section 3 .

\section{Threshold corrections to the gauge couplings for orbifold string vacua}

The starting point for calculating the one-loop threshold corrections $\triangle_{a}$ to the inverse gauge coupling $g_{a}^{-2}$ for orbifold compactifications is the general formula of [7], valid for any four- 
dimensional tachyon-free vacuum of the heterotic string

$$
\triangle_{a}=\int_{\mathcal{F}} \frac{d^{2} \tau}{\tau_{2}}\left[\mathcal{B}_{a}(\tau, \bar{\tau})-b_{a}\right]
$$

where

$$
\begin{aligned}
& \mathcal{B}_{a}(\tau, \bar{\tau})=2|\eta(\tau)|^{-4} \sum_{\text {even } s}(-1)^{s_{1}+s_{2}} \frac{\mathrm{d} Z_{\psi}(\mathbf{s}, \bar{\tau})}{2 \pi i \mathrm{~d} \bar{\tau}} \operatorname{tr}_{s_{1}}\left[Q_{a}^{2}(-1)^{s_{2} F} q^{H-\frac{11}{12}} \bar{q}^{\bar{H}-\frac{3}{8}}\right]_{\mathrm{int}}, \\
& b_{a}=\lim _{\tau_{2} \rightarrow \infty} \mathcal{B}_{a}(\tau, \bar{\tau})=-\frac{11}{3} \operatorname{tr}_{\mathrm{V}} Q_{a}^{2}+\frac{2}{3} \operatorname{tr}_{\mathrm{F}} Q_{a}^{2}+\frac{1}{3} \operatorname{tr}_{\mathrm{S}} Q_{a}^{2} .
\end{aligned}
$$

Here $\tau=\tau_{1}+i \tau_{2}$ is the modulus of the world-sheet torus and $\mathcal{F}$ is the fundamental region $\mathcal{F}=\left\{\tau \in \mathbb{C}\left|\tau_{2}>0,\right| \tau_{1}\left|<\frac{1}{2},\right| \tau \mid>1\right\}$. Furthermore $q=e^{2 \pi i \tau}$ and $Q_{a}$ is the charge of a state with respect to a generator of the gauge group labeled by $a$. The spin structure of the fermions is denoted by $\mathbf{s}=\left(s_{1}, s_{2}\right)$, where $s_{1}, s_{2} \in\{0,1\}$. A zero (one) refers to anti-periodic (periodic) boundary condition on the torus. The partition function for one complex fermion is given by

$$
Z_{\psi}(\mathbf{s}, \bar{\tau})=\frac{1}{\eta(\bar{\tau})} \theta\left[\begin{array}{l}
s_{2} / 2 \\
s_{1} / 2
\end{array}\right]
$$

The sum over the spin structures excludes the parity odd contribution $\mathbf{s}=(1,1)$. Finally $b_{a}$ is related to the one-loop $\beta$-function via $\beta_{a}=b_{a} g_{a}^{3} / 16 \pi^{2}$.

In ref. [12] the dependence of $\triangle_{a}$ on the internal moduli has been evaluated for certain orbifold compactifications. Such vacua are obtained [2] by moding the six-dimensional torus $T^{6}=\mathbb{R}^{6} / \Lambda$ of the compactification manifold by a twist $\Theta$ of finite order $\left(\Theta^{N}=1\right.$ for some $\left.N\right)$; in addition $\Theta$ has to be an automorphism of the lattice $\Lambda$. In refs. [7, 12] it was argued that the moduli dependence of the threshold corrections (2.2) arises only from the orbifold sectors with $\mathrm{N}=2$ space-time supersymmetry. Therefore we will not consider the completely untwisted sector with $\mathrm{N}=4$ space-time supersymmetry as well as $\mathrm{N}=1$ supersymmetry preserving sectors. The orbit of these $\mathrm{N}=2$ sectors will be denoted by $\mathcal{O}$ and is represented by the relevant set of twisted boundary conditions along the two cycles of the world-sheet torus. One property of $\mathrm{N}=2$ sectors is that they always have a complex plane left fixed by the orbifold twist $\Theta$. For calculational reasons it was assumed in ref. [12] that these fixed planes lie in a two dimensional even and self-dual sublattice $\Lambda_{2}$ of the full torus lattice $\Lambda$. Therefore their results can be applied only to models where the torus lattices $\Lambda$ can be written as an orthogonal decomposition of $\Lambda$ into a four dimensional lattice $\Lambda_{4}$ and a two dimensional lattice $\Lambda_{2}$, with the fixed plane lying in $\Lambda_{2}$. On the other hand there are a lot of orbifold models which do not fulfill this condition [20]; in the following we present the calculation of the threshold corrections for this larger class of orbifold models.

In ref. [12] only the difference $\triangle_{a_{1}} / k_{a_{1}}-\triangle_{a_{2}} / k_{a_{2}}$ between two gauge groups was calculated to get rid off the non-modular invariant regulator terms. In particular the difference $\left(\tau_{2} / k_{a_{1}}\right) \mathcal{B}_{a_{1}}-\left(\tau_{2} / k_{a_{2}}\right) \mathcal{B}_{a_{2}}$ is invariant under transformations of the modular group $\Gamma$ acting on $\tau$ with generators $S: \tau \rightarrow-1 / \tau$ and $T: \tau \rightarrow \tau+1$. Moreover it is easy to realize that the $\mathrm{N}=2$ part of the above difference is separately invariant under $\Gamma$ : this is because the generators $S$ and $T$ change the boundary conditions $\left(\Theta^{m}, \Theta^{n}\right)$ of a orbifold sector by $\left(\Theta^{m}, \Theta^{n}\right) \rightarrow\left(\Theta^{n}, \Theta^{N-m}\right)$ and $\left(\Theta^{m}, \Theta^{n}\right) \rightarrow\left(\Theta^{m}, \Theta^{m+n}\right)$, respectively. The $\mathrm{N}=2$ orbit $\mathcal{O}$ can be created from so-called fundamental elements by acting on them with modular transformations. The set of fundamental elements will be denoted by $\mathcal{O}_{0}$. Instead of integrating $\left(\tau_{2} / k_{a_{1}}\right) \mathcal{B}_{a_{1}}-\left(\tau_{2} / k_{a_{2}}\right) \mathcal{B}_{a_{2}}$ over the integration region $\mathcal{F}$ as in (2.1), the sum over all $\mathrm{N}=2$ sectors $\mathcal{O}$ can be replaced by the sum over the fundamental elements $\mathcal{O}_{0}$, provided that the integration region is extended to a region $\tilde{\mathcal{F}}$. Here $\tilde{\mathcal{F}}$ is generated by acting on $\mathcal{F}$ with exactly those modular transformations which generate $\mathcal{O}$ 
from $\mathcal{O}_{0}$. The integrand is then invariant only under a congruence subgroup $\Gamma_{0}$ of $\Gamma$ [21, 22] and $\tilde{\mathcal{F}}$ is its fundamental region. As an example consider the $Z_{4}$ orbifold generated by a twist with complex eigenvalues $\Theta=(i, i,-1)$. The three $\mathrm{N}=2$ sectors are $\mathcal{F}=\left\{\left(1, \Theta^{2}\right),\left(\Theta^{2}, 1\right),\left(\Theta^{2}, \Theta^{2}\right)\right\}$. All these sectors can be generated from the fundamental element $\left(1, \Theta^{2}\right)$ by an $\mathrm{S}-$ and an STtransformations, respectively. The appropriate extension of the integration region is then given by $\tilde{\mathcal{F}}=\{1, S, S T\} \mathcal{F}$.

In general there is no known way of calculating integrals over the fundamental region of modular functions analytically. Fortunately the $\tau$-integration over $\left(\tau_{2} / k_{a_{1}}\right) \mathcal{B}_{0 a_{1}}-\left(\tau_{2} / k_{a_{2}}\right) \mathcal{B}_{0 a_{2}}$ I simplifies to an integration only over the zero mode part $Z_{(g, h)}^{\text {torus }}(\tau, \bar{\tau})$ corresponding to the winding and Kaluza-Klein modes of the fixed torus. Here $(g, h)$ denotes the dependence of this function on the orbifold sector; we will give an explicit expression below. This result can be seen in two different ways: first one can consider the explicit representation of the integrand in terms of $\theta$-functions. The charge operators in the trace over the internal part can be represented by $\tau$-derivatives on the appropriate $\theta$-functions of the gauge sector [23]. Indeed the derivative of $Z_{\psi}$ in (2.2) has a similar source. In this way the integrand splits into two parts: first $Z_{(g, h)}^{\text {torus }}(\tau, \bar{\tau})$ representing the zero-modes of that part of the lattice which is left unrotated by the twist. This part contains the full moduli dependence and multiplied by $\tau_{2}$ it is invariant under a subgroup $\Gamma_{0}$ of $\Gamma$. The other part describes the rest of the right-moving and the left-moving sector of a string twisted by $(g, h)$. It is separately invariant under $\Gamma_{0}$ and bounded in the fundamental region $\tilde{\mathcal{F}}$ of $\Gamma_{0}$. For the relevant subgroups the only automorphic functions without singularities in the whole fundamental region are constants due to general theorems 21]. What will remain is therefore an integral over the zero-mode parts $Z_{\left(g_{0}, h_{0}\right)}^{\text {torus }}(\tau, \bar{\tau})$, where $\left(g_{0}, h_{0}\right) \in \mathcal{O}_{0}$, with integration region $\tilde{\mathcal{F}}$ multiplied by a constant expression to be determined.

Actually there is a second more elegant way to derive this result, namely a generalization of the method of ref. [12]. The main difference is that $Z_{(g, h)}^{\text {torus }}(\tau, \bar{\tau})$ depends now on the orbifold sector $(g, h)$, in contrast to the orbifold models considered in [12]. The $\mathrm{N}=2$ orbit of interest can be interpreted as an $\mathrm{N}=2$ supersymmetric model in four dimensions. This is because this orbit itself would produce an orbifold with $\mathrm{N}=2$ space-time supersymmetry. The internal part of a theory with an $\mathrm{N}=2$ spacetime supersymmetry splits into one piece with an $\mathrm{N}=2$ and a second piece with an $\mathrm{N}=4$ superconformal symmetry [24]. Moreover it couples to the spin structure of the world-sheet fermions only via an $S U(2)$ current [24]. This fact can be used to rewrite $\mathcal{B}_{a}(\tau, \bar{\tau})$ of $(2.2)$ as follows:

$$
\begin{aligned}
\mathcal{B}_{a}(\tau, \bar{\tau}) & =\sum_{(g, h) \in \mathcal{O}} Z_{(g, h)}^{\text {torus }}(\tau, \bar{\tau}) \mathcal{C}_{a}^{(g, h)}(\tau), \\
\mathcal{C}_{a}^{(g, h)}(\tau) & =\eta^{-4}(\tau) \operatorname{tr}_{R}\left[\frac{1}{2}(-1)^{\mathrm{F}} Q_{a}^{2} q^{H-\frac{5}{6}} \bar{q}^{\bar{H}-\frac{1}{4}}\right]_{\substack{(g, \bar{c})=(20,6) \\
(c, h)}} .
\end{aligned}
$$

Here $\mathcal{C}_{a}^{(g, h)}(\tau)$ contains the $E_{8} \times E_{8}$ current algebra twisted by $(g, h)$, the contributions of the four internal twisted dimensions, the oscillator part of the fixed torus and the contribution of the four space-time dimensions.

Now $\tau_{2} Z_{\left(g_{0}, h_{0}\right)}^{\text {torus }}(\tau, \bar{\tau})$ is invariant under $\Gamma_{0}$-transformations on $\tau$.Since $\left(\tau_{2} / k_{a_{1}}\right) \mathcal{B}_{a_{1}}-\left(\tau_{2} / k_{a_{2}}\right) \mathcal{B}_{a_{2}}$ is invariant under $\Gamma$ and therefore in particular under $\Gamma_{0}$, we can follow that the function $1 / k_{a_{1}} \mathcal{C}_{a_{1}}^{\left(g_{0}, h_{0}\right)}(\tau)-1 / k_{a_{2}} \mathcal{C}_{a_{2}}^{\left(g_{0}, h_{0}\right)}(\tau)$ has to be invariant under $\Gamma_{0}$ as well. Moreover it is nowhere singular as can be seen from the limit $\tau_{2} \rightarrow \infty$. Therefore it has to be a constant by the virtue of the above mentioned theorems. This constant represents the contribution $b_{a}^{(g, h)}$ of the sector $(g, h)$ to the one-loop $\beta$-function coefficient $b_{a}$. Including an infrared regularization [7], the threshold corrections (2.2) can be expressed by the following integral:

\footnotetext{
${ }^{1}$ The zero of $\mathcal{B}_{0}$ means that the trace in $(2.2)$ is restricted to $\mathcal{O}_{0}$.
} 


$$
\begin{aligned}
\triangle_{a} & =\int_{\mathcal{F}} \frac{d^{2} \tau}{\tau_{2}}\left[\sum_{(g, h) \in \mathcal{O}} \frac{Z_{(g, h)}^{\text {torus }}(\tau, \bar{\tau})}{\lim _{\tau_{2}^{\prime} \rightarrow \infty} Z_{(g, h)}^{\text {torus }}\left(\tau^{\prime}, \bar{\tau}^{\prime}\right)} b_{a}^{(g, h)}-b_{a}^{N=2}\right] \\
& =\sum_{\left(g_{0}, h_{0}\right) \in \mathcal{O}_{0}} b_{a}^{\left(g_{0}, h_{0}\right)} \int_{\tilde{\mathcal{F}}} \frac{d^{2} \tau}{\tau_{2}} Z_{\left(g_{0}, h_{0}\right)}^{\text {torus }}(\tau, \bar{\tau})-b_{a}^{N=2} \int_{\mathcal{F}} \frac{d^{2} \tau}{\tau_{2}} .
\end{aligned}
$$

Here $b_{a}^{N=2}$ denotes the $\beta$-function coefficient arising from the $\mathrm{N}=2$ sectors of the $Z_{N}$ orbifold under consideration and we have used the identity $\lim _{\tau_{2} \rightarrow \infty} Z_{\left(g_{0}, h_{0}\right)}^{\text {torus }}\left(\tau, \bar{\tau}^{\prime}\right)=1$. The first integral on the r.h.s represents a Petersson product on the space of modular functions of the corresponding subgroup [22, 25].

\subsection{Threshold corrections for the $S U(4)^{2} / Z_{4}$ orbifold}

In the rest of this section we will apply the previous analysis to some orbifold models which can not be treated with the method of ref. [12]. First we consider the $Z_{4}$ orbifold defined by two Coxeter twists in two root lattices of $S U(4)$ and deformations of them. The twist $Q$ with the complex eigenvalues $\Theta=(i, i,-1)$ can be represented w.r.t to the lattice basis $\left(e_{i}^{\prime}=Q_{i}{ }^{j} e_{j}\right)$ by the matrix:

$$
Q=\left(\begin{array}{cccccc}
0 & 0 & -1 & 0 & 0 & 0 \\
1 & 0 & -1 & 0 & 0 & 0 \\
0 & 1 & -1 & 0 & 0 & 0 \\
0 & 0 & 0 & 0 & 0 & -1 \\
0 & 0 & 0 & 1 & 0 & -1 \\
0 & 0 & 0 & 0 & 1 & -1
\end{array}\right)
$$

There are six complex untwisted moduli: five $(1,1)$-moduli and one $(2,1)$-modulus due to the five untwisted $\overline{\mathbf{2 7}}$ generations and one untwisted $\mathbf{2 7}$ generation [2, 20]. Therefore the metric $g$ (defined by $g_{i j}=<e_{i} \mid e_{j}>$ ) has seven and the antisymmetric tensor field $b$ five real deformations. The equations $g Q=Q^{*} g$ and $b Q=Q^{*} b$ determine the background fields in terms of the independent deformation parameter: [26, 27]. Solving these equations one obtains

$$
g=\left(\begin{array}{cccccc}
R^{2} & x & -R^{2}-2 x & u & v & -u-v-z \\
x & R^{2} & x & z & u & v \\
-R^{2}-2 x & x & R^{2} & -u-v-z & z & u \\
u & z & -u-v-z & S^{2} & y & -S^{2}-2 y \\
v & u & z & y & S^{2} & y \\
-u-v-z & v & u & -S^{2}-2 y & y & S^{2}
\end{array}\right)
$$

with $R, S, x, y, u, v, z \in \mathbb{R}$ and

$$
b=\left(\begin{array}{cccccc}
0 & \alpha & 0 & \gamma & \delta & -\gamma-\delta-\epsilon \\
-\alpha & 0 & \alpha & \epsilon & \gamma & \delta \\
0 & -\alpha & 0 & -\gamma-\delta-\epsilon & \epsilon & \gamma \\
-\gamma & -\epsilon & \gamma+\delta+\epsilon & 0 & \beta & 0 \\
-\delta & -\gamma & -\epsilon & -\beta & 0 & \beta \\
\gamma+\delta+\epsilon & -\delta & -\gamma & 0 & -\beta & 0
\end{array}\right)
$$

with $\alpha, \beta, \gamma, \delta, \epsilon \in \mathbb{R}$.

\footnotetext{
${ }^{2}$ We do not consider the more general case with a non-commuting $b$ field 27].
} 
As mentioned previously the $\mathrm{N}=2$ orbit is given by $\mathcal{O}=\left\{\left(1, \Theta^{2}\right),\left(\Theta^{2}, 1\right),\left(\Theta^{2}, \Theta^{2}\right)\right\}$. $\left(\Theta^{2}, 1\right)$ can be obtained from the fundamental element $\left(1, \Theta^{2}\right)$ by an $S$-transformation on $\tau$ and similarly $\left(\Theta^{2}, \Theta^{2}\right)$ by an $S T$-transformation. The zero mode parts $Z_{(g, h)}^{\text {torus }}$ of the fixed plane take the following form [20]:

$$
\begin{aligned}
& Z_{\left(1, \Theta^{2}\right)}^{\text {torus }}(\tau, \bar{\tau}, g, b)=\sum_{P \in \Lambda_{N}^{\perp}} q^{\frac{1}{2} P_{L}^{2}} \bar{q}^{\frac{1}{2} P_{R}^{2}} \\
& Z_{\left(\Theta^{2}, 1\right)}^{\text {torus }}(\tau, \bar{\tau}, g, b)=\frac{1}{V_{\Lambda_{N}^{\perp}}} \sum_{P \in\left(\Lambda_{N}^{\perp}\right)^{*}} q^{\frac{1}{2} P_{L}^{2}} \bar{q}^{\frac{1}{2} P_{R}^{2}}, \\
& Z_{\left(\Theta^{2}, \Theta^{2}\right)}^{\text {torus }}(\tau, \bar{\tau}, g, b)=\frac{1}{V_{\Lambda_{N}^{\perp}}} \sum_{P \in\left(\Lambda_{N}^{\perp}\right)^{*}} e^{\pi i\left(P_{L}^{2}-P_{R}^{2}\right)} q^{\frac{1}{2} P_{L}^{2}} \bar{q}^{\frac{1}{2} P_{R}^{2}} .
\end{aligned}
$$

Here $\Lambda_{N}$ denotes the Narain lattice of $S U(4)^{2}$ with momentum vectors [28]

$$
P_{L}=\frac{p}{2}+(g-b) w, \quad P_{R}=\frac{p}{2}-(g+b) w .
$$

$\Lambda_{N}^{\perp}$ is that part of the lattice which remains fixed under $Q^{2}$ and $V_{\Lambda_{N}}$ is the volume of this sublattice. It is worth mentioning that the partition functions $Z_{(g, h)}^{\text {torus }}(\tau, \bar{\tau}, g, b)$ of ref. [12] are independent of the orbifold sector $(g, h)$ because there $\Lambda_{N}^{\perp}$ is an even self-dual sublattice. As a consequence $\tau_{2} Z_{(g, h)}^{\text {torus }}(\tau, \bar{\tau}, g, b)$ was invariant under $\Gamma$ in contrast to the functions of eq. (2.9).

The subspace corresponding to $\Lambda_{N}^{\perp}$ can be described by the following winding and momentum vectors, respectively:

$$
w=\left(\begin{array}{c}
n^{1} \\
0 \\
n^{1} \\
n^{2} \\
0 \\
n^{2}
\end{array}\right), n^{1}, n^{2} \in \mathbb{Z} \quad \text { and } p=\left(\begin{array}{c}
m_{1} \\
-m_{1} \\
m_{1} \\
m_{2} \\
-m_{2} \\
m_{2}
\end{array}\right), m_{1}, m_{2} \in \mathbb{Z} .
$$

They are determined by the equations $Q^{2} w=w$ and $Q^{*^{2}} p=p$. The partition function $\tau_{2} Z_{\left(1, \Theta^{2}\right)}^{\text {torus }}(\tau, \bar{\tau}, g, b)$ is invariant under the group $\Gamma_{0}(2)$ which belongs to the congruence subgroups of $\Gamma$ [21]. $\Gamma_{0}(2)$ is generated by the elements $T$ and $S T^{2} S$ of $\Gamma$. In general the group $\Gamma_{0}(n)$ can be represented by the following set of two by two matrices acting on $\tau$ as $\tau \rightarrow \frac{a \tau+b}{c \tau+d}$ :

$$
\Gamma_{0}(n)=\left\{\left(\begin{array}{ll}
a & b \\
c & d
\end{array}\right) \mid a d-b c=1, c=0 \bmod n\right\} .
$$

Instead of integrating the contribution of the various sectors $(g, h)$ over the fundamental region $\mathcal{F}$, we integrate only the contribution $Z_{\left(1, \Theta^{2}\right)}^{\text {torus }}$ but over the extended integration region $\mathcal{F}=\{1, S, S T\} \mathcal{F}\left(\right.$ cf. (2.5)). The explicit expression for $Z_{\left(1, \Theta^{2}\right)}^{\text {torus }}$ can be determined with (2.11) to be

$$
\begin{aligned}
Z_{\left(1, \Theta^{2}\right)}^{\text {torus }}(\tau, \bar{\tau}, g, b) & =\sum_{\left(P_{L}, P_{R}\right) \in \Lambda_{N}^{\perp}} q^{\frac{1}{2} P_{L}^{t} g^{-1} P_{L}} \bar{q}^{\frac{1}{2} P_{R}^{t} g^{-1} P_{R}} \\
& =\sum_{p, w} e^{2 \pi i \tau p^{t} w} e^{-\pi \tau_{2}\left(\frac{1}{2} p^{t} g^{-1} p-2 p^{t} g^{-1} b w+2 w^{t} g w-2 w^{t} b g^{-1} b w-2 p^{t} w\right)}
\end{aligned}
$$


The last expression can be written in terms of the $(1,1)$-modulus $T$ and the $(2,1)$-modulus $U$ corresponding to $\partial Y^{3}(z)$ and $\bar{\partial} Y^{3}(\bar{z})$ [29]. They are defined by:

$$
T=T_{1}+i T_{2}=2\left(b+i \sqrt{\operatorname{det} g_{\perp}}\right) \quad, \quad U=U_{1}+i U_{2}=\frac{1}{g_{\perp 11}}\left(g_{\perp 12}+\sqrt{\operatorname{det} g_{\perp}}\right) .
$$

Here $g_{\perp}$ is uniquely determined by $w^{t} g w=\left(n^{1} n^{2}\right) g_{\perp}\left(\begin{array}{l}n^{1} \\ n^{2}\end{array}\right)$. This way one gets

$$
\begin{aligned}
T & =4\left[-\delta-\epsilon+i \sqrt{4 x y-(v+z)^{2}}\right], \\
U & =\frac{1}{2 x}\left[v+z-i \sqrt{4 x y-(v+z)^{2}}\right] .
\end{aligned}
$$

This definition of $T$ and $U$ agrees with that appearing in the Kähler potential [10, 6]. The partition function $Z_{\left(1, \Theta^{2}\right)}^{\text {torus }}(\tau, \bar{\tau}, g, b)$ takes now the form

$$
Z_{\left(1, \Theta^{2}\right)}^{\text {torus }}(\tau, \bar{\tau}, T, U)=\sum_{\substack{m_{1}, m_{2} \in 2 \mathbb{Z} \\ n^{1}, n^{2} \in \mathbb{Z}}} e^{2 \pi i \tau\left(m_{1} n^{1}+m_{2} n^{2}\right)} e^{\frac{-\pi \tau_{2}}{T_{2} U_{2}}\left|T U n^{2}+T n^{1}-U m_{1}+m_{2}\right|^{2}} .
$$

This expression can be rewritten performing a Poisson resummation on $m_{1}$ and $m_{2}$ :

$$
\tau_{2} Z_{\left(1, \Theta^{2}\right)}^{\text {torus }}(\tau, \bar{\tau}, T, U)=\frac{1}{4} \sum_{A \in \mathcal{M}} e^{-2 \pi i T \operatorname{det} A} T_{2} e^{\frac{-\pi T_{2}}{\tau_{2} U_{2}}\left|(1, U) A\left(\begin{array}{c}
\tau \\
1
\end{array}\right)\right|^{2}},
$$

where

$$
\mathcal{M}=\left\{\left(\begin{array}{cc}
n_{1} & \frac{1}{2} l_{1} \\
n_{2} & \frac{1}{2} l_{2}
\end{array}\right) \mid n_{1}, n_{2}, l_{1}, l_{2} \in \mathbb{Z}\right\} .
$$

At this point we want to stress that the set $\mathcal{M}$ is larger than that of ref. [12] due to the halfinteger entries in the right column. The restriction on even momentum numbers in $(2.16)$ is a direct consequence of the definitions (2.15). However it is important to note that there is no discrete transformation on the background [26, 27] which allows one to return to integer moded momentum and winding numbers.

From (2.17) one can obtain $\tau_{2} Z_{\left(\Theta^{2}, 1\right)}^{\text {torus }}(\tau, \bar{\tau})$ by an $S$-transformation on $\tau$. After exchanging $n_{i}$ and $l_{i}$ and performing again a Poisson resummation on $l_{i}$ one obtains

$$
Z_{\left(\Theta^{2}, 1\right)}^{\text {torus }}(\tau, \bar{\tau}, T, U)=\frac{1}{4} \sum_{\substack{m_{1}, m_{2} \in \mathbb{Z} \\ n^{1}, n^{2} \in \mathbb{Z}}} e^{2 \pi i \tau\left(m_{1} \frac{n^{1}}{2}+m_{2} \frac{n^{2}}{2}\right)} e^{\frac{-\pi \tau_{2}}{T_{2} U_{2}}\left|T U \frac{n^{2}}{2}+T \frac{n^{1}}{2}-U m_{1}+m_{2}\right|^{2}} .
$$

The factor $1 / 4$ can be identified with the volume of the dual sublattice in (2.9). This point has been discussed already in [20]. $\tau_{2} Z_{\left(\Theta^{2}, 1\right)}^{\text {torus }}(\tau, \bar{\tau}, T, U)$ is invariant under $\Gamma^{0}(2)$ acting on $\tau$ and is identical to that for the $\left(\Theta^{2}, \Theta^{2}\right)$ sector. $\Gamma^{0}(2)$ is defined similar to $(2.12)$ but with $b=0$ mod 2 instead of $c=0 \bmod 2$. Thus the contribution of the two sectors $\left(\Theta^{2}, 1\right)$ and $\left(\Theta^{2}, \Theta^{2}\right)$ to the coefficient $b_{a}^{N=2}$ of the $\beta$-function is one fourth of that of the sector $\left(1, \Theta^{2}\right)$ and we get

$$
b_{a}^{N=2}=\frac{3}{2} b_{a}^{\left(1, \Theta^{2}\right)} .
$$

The details of the integration of $Z_{\left(1, \Theta^{2}\right)}^{\text {torus }}$ over the modular parameter $\tau$ are given in the appendix. The final result for the threshold correction to the inverse gauge coupling (2.5) reads 


$$
\begin{aligned}
\triangle_{a}(T, \bar{T}, U, \bar{U})= & -b_{a}^{\left(1, \Theta^{2}\right)} \ln \left[\frac{8 \pi e^{1-\gamma_{E}}}{3 \sqrt{3}} \frac{T_{2}}{4}\left|\eta\left(\frac{T}{2}\right)\right|^{4} U_{2}|\eta(U)|^{4}\right] \\
& -\frac{1}{2} b_{a}^{\left(1, \Theta^{2}\right)} \ln \left[\frac{8 \pi e^{1-\gamma_{E}}}{3 \sqrt{3}} T_{2}\left|\eta\left(\frac{T}{2}\right)\right|^{4} U_{2}|\eta(U)|^{4}\right] .
\end{aligned}
$$

Formula (2.20) has the following interesting properties. First the non-holomorphic moduli dependent part corresponding to massless modes running in the loops agrees with that of ref. 12] up to the modified $\beta$-function coefficient (2.19). Note that $b_{a}^{N=2}$ depends on the torus lattice [20]. The functional dependence of the non-holomorphic part was expected since it determines the field theoretical anomaly w.r.t. modular transformations on $T$ and should be independent of the torus lattice. The second point is the symmetry of eq. (2.20) w.r.t. transformations on the moduli $T$ and $U$. Whereas the Kähler potentials [10, 6] are invariant under a symmetry $\Gamma_{T} \times \Gamma_{U}$ acting on $T$ and $U$, eq. (2.20) is only invariant under the subgroup $\Gamma^{0}(2)_{T} \times \Gamma_{U}$.

It is helpful to consider the contribution of the relevant two-dimensional untwisted subspace to the energy of an unexcited string twisted in the remaining dimensions. Because of twist invariance the momenta and windings w.r.t. to the other dimensions vanish and we get

$$
m_{\perp}^{2}=\sum_{\substack{m_{1}, m_{2} \in \mathbb{Z} \\ n^{1}, n^{2} \in \mathbb{Z}}} \frac{1}{T_{2} U_{2}}\left|T U n^{2}+T n^{1}-2 U m_{1}+2 m_{2}\right|^{2} .
$$

The common belief is that such a formula should be invariant under the symmetry $\Gamma_{T} \times \Gamma_{U}$ acting on $T$ and $U$ respectively. But it is not hard to see that the above expression allows only the following transformations on $T$ and $U$ :

$$
\begin{gathered}
T \rightarrow T+2 \quad, \quad T \rightarrow \frac{T}{T+1}, \\
U \longrightarrow U+1 \quad, \quad U \rightarrow-\frac{1}{U} .
\end{gathered}
$$

These are the generators of the group $\Gamma^{0}(2)_{T}$ acting on $T$ and $\Gamma_{U}$ acting on $U$, respectively. A general $\Gamma$ transformation on $T$ represented by $T \rightarrow \frac{a T+b}{c T+d}$ requires the following redefinition of the windings and momenta:

$$
\left(\begin{array}{c}
n^{\prime 1} \\
n^{\prime 2} \\
m_{1}^{\prime} \\
m_{2}^{\prime}
\end{array}\right)=\left(\begin{array}{cccc}
a & 0 & 0 & 2 c \\
0 & a & -2 c & 0 \\
0 & -b / 2 & d & 0 \\
b / 2 & 0 & 0 & d
\end{array}\right)\left(\begin{array}{c}
n^{1} \\
n^{2} \\
m_{1} \\
m_{2}
\end{array}\right) .
$$

From this expression it follows immediately that $b$ has to be an even number. Note that under the above unimodular transformation the spin $\left(P_{L}^{2}-P_{R}^{2}\right)_{\perp}$ remains invariant too. These results should be compared with the results obtained in [29, 30] for two-dimensional compactifications on Narain lattices.

Expression (2.21) can be used to consider the topological free energy of the subspace defined by $\Lambda_{\perp}$ [19]:

$$
F(T, \bar{T}, U, \bar{U})=\sum_{\substack{m_{1}, m_{2} \in \mathbb{Z} \\ n^{1}, n^{2} \in \mathbb{Z}}} \ln \frac{\left|T U n^{2}+T n^{1}-2 U m_{1}+2 m_{2}\right|^{2}}{T_{2} U_{2}} .
$$

$F$ is the contribution of the two-dimensional unrotated subspace to the total free energy. Since $F$ is defined to be a topological quantity the sum of (2.23) does not run over oscillator excitations 
and is therefore subject to the constraint $P_{L}^{2}-P_{R}^{2}=4\left(m_{1} n_{1}+m_{2} n_{2}\right)=0$. This equation can be solved in two inequivalent orbits [18]. Proceeding similar as in [19] we arrive at

$$
F(T, \bar{T}, U, \bar{U})=\ln \left[\frac{T_{2}}{4}\left|\eta\left(\frac{T}{2}\right)\right|^{4} U_{2}|\eta(U)|^{4}\right] .
$$

Interestingly the topological free energy for $\left(\Lambda_{\perp}\right)^{*}$ does not agree with (2.24). It can be evaluated from (2.18) to be

$$
\tilde{F}(T, \bar{T}, U, \bar{U})=\frac{1}{2} \ln \left[T_{2}\left|\eta\left(\frac{T}{2}\right)\right|^{4} U_{2}|\eta(U)|^{4}\right] .
$$

Both results together agree with 2.20$)$ up to the factor $b_{a}^{\left(1, \Theta^{2}\right)}$.

\subsection{Threshold corrections for the $S U(3) \times S O(8) / Z_{6}$ orbifold}

Next we investigate a $Z_{6}$ orbifold with torus lattice $S U(3) \times S O(8)$. The complex twist is given by $\Theta=\exp \left[\frac{2 \pi i}{6}(2,1,-3)\right]$. Therefore the twists $\Theta^{2}$ and $\Theta^{4}$ leave invariant the third complex subspace which lies in the $S O(8)$ lattice. There is also a fixed plane for $\Theta^{3}$. Since this plane lies in the first two coordinates which correspond to the $S U(3)$ lattice, the results of [12] can be applied and we will consider its contribution to the thresholds only at the end. The twist w.r.t the lattice basis is defined to be

$$
Q=\left(\begin{array}{cccccc}
0 & -1 & 0 & 0 & 0 & 0 \\
1 & -1 & 0 & 0 & 0 & 0 \\
0 & 0 & 0 & 1 & -1 & -1 \\
0 & 0 & 1 & 1 & -1 & -1 \\
0 & 0 & 0 & 1 & -1 & 0 \\
0 & 0 & 0 & 1 & 0 & -1
\end{array}\right)
$$

The $Q^{2}$ and $Q^{*^{2}}$ invariant subplanes can be parameterized by the following vectors, respectively:

$$
w=\left(\begin{array}{c}
0 \\
0 \\
n^{1} \\
0 \\
n^{1}-n^{2} \\
n^{2}
\end{array}\right), n^{1}, n^{2} \in \mathbb{Z} \quad \text { and } \quad p=\left(\begin{array}{c}
0 \\
0 \\
m_{1} \\
-m_{1} \\
m_{2} \\
m_{1}-m_{2}
\end{array}\right), \quad m_{1}, m_{2} \in \mathbb{Z}
$$

Proceeding similar as before one obtains for the metric and antisymmetric background fields in the lattice basis

$$
g=\left(\begin{array}{cccccc}
R^{2} & -R^{2} / 2 & 0 & 0 & 0 & 0 \\
-R^{2} / 2 & R^{2} & 0 & 0 & 0 & 0 \\
0 & 0 & S^{2} & x & z & -S^{2}-2 x-z \\
0 & 0 & x & S^{2} & -S^{2}-x-z & x+z \\
0 & 0 & z & -S^{2}-x-z & V^{2} & 2 S^{2}-V^{2}+2 x+z \\
0 & 0 & -S^{2}-2 x-z & x+z & 2 S^{2}-V^{2}+2 x+z & -S^{2}+V^{2}-2 x-2 z
\end{array}\right)
$$


with $R, S, V, x, z \in \mathbb{R}$ and

$$
b=\left(\begin{array}{cccccc}
0 & \alpha & 0 & 0 & 0 & 0 \\
-\alpha & 0 & 0 & 0 & 0 & 0 \\
0 & 0 & 0 & \beta & -\gamma & \gamma \\
0 & 0 & -\beta & 0 & \beta+\gamma & \beta-\gamma \\
0 & 0 & \gamma & -\beta-\gamma & 0 & \gamma \\
0 & 0 & -\gamma & -\beta+\gamma & -\gamma & 0
\end{array}\right), \quad \alpha, \beta, \gamma \in \mathbb{R}
$$

For the moduli corresponding to the third complex plane we get

$$
\begin{aligned}
U & =\frac{1}{S^{2}+V^{2}+2 z}\left[S^{2}-2 V^{2}-z+i \sqrt{\operatorname{det} g_{\perp}}\right], \\
T & =2\left[3 \gamma+i \sqrt{\operatorname{det} g_{\perp}}\right]
\end{aligned}
$$

where

$$
\operatorname{det} g_{\perp}:=3 S^{2} V^{2}-6 S^{4}-6 S^{2} x-6 V^{2} x-12 S^{2} z-12 x z-9 z^{2} .
$$

The $\mathrm{N}=2$ orbit is $\mathcal{O}=\left\{\left(1, \Theta^{2}\right),\left(\Theta^{2}, 1\right),\left(\Theta^{2}, \Theta^{2}\right),\left(\Theta^{2}, \Theta^{4}\right),\left(1, \Theta^{4}\right),\left(\Theta^{4}, 1\right),\left(\Theta^{4}, \Theta^{4}\right),\left(\Theta^{4}, \Theta^{2}\right)\right\}$. The first four sectors are the CPT conjugate of the following ones. The fundamental orbit $\mathcal{O}_{0}$ consists of $\left(1, \Theta^{2}\right)$ and $\left(1, \Theta^{4}\right)$. All the remaining elements of $\mathcal{O}$ can be generated by acting on these two sectors with the group elements $S, S T$ and $S T^{2}$. The sum $\tau_{2} Z_{\left(1, \Theta^{2}\right)}^{\text {torus }}(\tau, \bar{\tau})+\tau_{2} Z_{\left(1, \Theta^{4}\right)}^{\text {torus }}(\tau, \bar{\tau})$ is invariant under the group $\Gamma_{0}(3)$ generated by $T$ and $S T^{3} S$. Its fundamental region $\tilde{\mathcal{F}}$ is given by the set $\tilde{\mathcal{F}}=\left\{1, S, S T, S T^{2}\right\} \mathcal{F}[21]$. Acting on $\tau$ with an $S T^{3} S$-transformation one can show that $Z_{\left(1, \Theta^{4}\right)}^{\text {torus }}(\tau, \bar{\tau})=Z_{\left(1, \Theta^{2}\right)}^{\text {torus }}(\tau, \bar{\tau})$ and thus it is sufficient to integrate $2 Z_{\left(1, \Theta^{2}\right)}^{\text {torus }}(\tau, \bar{\tau})$ over the region $\tilde{\mathcal{F}}$. From $(2.9)$ and $(2.26)$ we find

$$
Z_{\left(1, \Theta^{2}\right)}^{\text {torus }}(\tau, \bar{\tau}, T, U)=\sum_{\substack{m_{1}, m_{2} \in \mathbb{Z} \\ n^{1}, n^{2} \in \mathbb{Z}}} e^{2 \pi i \tau\left(m_{1} n_{1}+m_{1} n_{2}+m_{2} n_{1}-2 m_{2} n_{2}\right)} e^{\frac{-\pi \tau_{2}}{T_{2} U_{2}}\left|T U n^{2}+T n^{1}-U\left(m_{1}+m_{2}\right)+m_{1}-2 m_{2}\right|^{2}}
$$

After a Poisson transformation in the momenta and a special linear transformation eq. (2.28) becomes

$$
\tau_{2} Z_{\left(1, \Theta^{2}\right)}^{\text {torus }}(\tau, \bar{\tau}, T, U)=\frac{1}{3} \sum_{A \in \mathcal{M}} e^{-2 \pi i T \operatorname{det} A} T_{2} e^{\frac{-\pi T_{2}}{\tau_{2} U_{2}^{\prime}}\left|\left(1, U^{\prime}\right) A\left(\begin{array}{c}
\tau \\
1
\end{array}\right)\right|^{2}}
$$

where

$$
\mathcal{M}=\left\{\left(\begin{array}{cc}
n_{1} & l_{1} \\
n_{2} & \frac{1}{3} l_{2}
\end{array}\right) \mid n_{1}, n_{2}, l_{1}, l_{2} \in \mathbb{Z}\right\}
$$

and $U^{\prime}=U+2$. Since the coefficients $b_{a}^{\left(1, \Theta^{2}\right)}$ and $b_{a}^{\left(1, \Theta^{4}\right)}$ are three times larger than those of the remaining $\mathrm{N}=2$ sectors we have

$$
b_{a}^{N=2}=4 b_{a}^{\left(1, \Theta^{2}\right)} .
$$

Again the details of the $\tau$-integration are given in the appendix. The final result for the threshold corrections (2.5) reads 


$$
\begin{aligned}
\triangle_{a}(T, \bar{T}, U, \bar{U})= & -2 b_{a}^{\left(1, \Theta^{2}\right)} \ln \left[\frac{8 \pi e^{1-\gamma_{E}}}{3 \sqrt{3}} \frac{T_{2}}{3}\left|\eta\left(\frac{T}{3}\right)\right|^{4} \frac{U_{2}}{3}\left|\eta\left(\frac{U+2}{3}\right)\right|^{4}\right] \\
& -2 b_{a}^{\left(1, \Theta^{2}\right)} \ln \left[\frac{8 \pi e^{1-\gamma_{E}}}{3 \sqrt{3}} T_{2}|\eta(T)|^{4} U_{2}|\eta(U+2)|^{4}\right] \\
& -\hat{b}_{a} \ln \left[\frac{8 \pi e^{1-\gamma_{E}}}{3 \sqrt{3}} \hat{T}_{2}|\eta(\hat{T})|^{4} \hat{U}_{2}|\eta(\hat{U})|^{4}\right] .
\end{aligned}
$$

The third term arises from the fixed plane in the lattice of $S U(3) . \hat{b}_{a}$ is the contribution of the sectors $\left(1, \Theta^{3}\right),\left(\Theta^{3}, 1\right)$ and $\left(\Theta^{3}, \Theta^{3}\right)$ to the coefficient of the $\beta$-function and $\hat{T}$ and $\hat{U}$ are the moduli corresponding to this plane.

Again we consider the spectrum of strings with non-vanishing winding and momentum numbers only in the relevant subspace:

$$
m_{\perp}^{2}=\sum_{\substack{m_{1}, m_{2} \in \mathbb{Z} \\ n^{1}, n^{2} \in \mathbb{Z}}} \frac{1}{T_{2} U_{2}^{\prime}}\left|T U^{\prime} n^{2}+T n^{1}-U^{\prime} m_{1}+3 m_{2}\right|^{2}
$$

Its symmetry group is given by $\Gamma^{0}(3)_{T} \times \Gamma^{0}(3)_{U^{\prime}}$ with generators

$$
\begin{gathered}
T \rightarrow T+3 \quad, \quad T \longrightarrow \frac{T}{T+1}, \\
U^{\prime} \longrightarrow U^{\prime}+3 \quad, \quad U^{\prime} \longrightarrow \frac{U^{\prime}}{U^{\prime}+1} .
\end{gathered}
$$

The above transformations accompanied by a proper unimodular transformation on the winding and momentum numbers keep invariant the spin $\left(P_{L}^{2}-P_{R}^{2}\right)_{\perp}$. The free energy of $\Lambda_{N}^{\perp}$ can be calculated to be

$$
F(T, \bar{T}, U, \bar{U})=\ln \left[\frac{T_{2}}{3}\left|\eta\left(\frac{T}{3}\right)\right|^{4} U_{2}|\eta(U+2)|^{4}\right]+\ln \left[T_{2}|\eta(T)|^{4} \frac{U_{2}}{3}\left|\eta\left(\frac{U+2}{3}\right)\right|^{4}\right]
$$

and similarly for $\left(\Lambda_{N}^{\perp}\right)^{*}$ :

$$
\tilde{F}(T, \bar{T}, U, \bar{U})=\ln \left[T_{2}|\eta(T)|^{4} U_{2}|\eta(U+2)|^{4}\right]+\ln \left[\frac{T_{2}}{3}\left|\eta\left(\frac{T}{3}\right)\right|^{4} \frac{U_{2}}{3}\left|\eta\left(\frac{U+2}{3}\right)\right|^{4}\right]
$$

Both results together agree with 2.31) up to the factor $b_{a}^{\left(1, \Theta^{2}\right)}$.

\subsection{Threshold corrections for the $S U(2) \times S O(10) / Z_{8}$ orbifold}

The $Z_{8}$-IIa orbifold with torus lattice $S U(2) \times S O(10)$ has the complex twist $\Theta=\exp \left[\frac{2 \pi i}{8}(1,3,-4)\right]$. W.r.t the lattice basis vectors the twist reads

$$
Q=\left(\begin{array}{cccccc}
0 & 0 & 1 & -1 & -1 & 0 \\
1 & 0 & 1 & -1 & -1 & 0 \\
0 & 1 & 1 & -1 & -1 & 0 \\
0 & 0 & 1 & -1 & 0 & 0 \\
0 & 0 & 1 & 0 & -1 & 0 \\
0 & 0 & 0 & 0 & 0 & -1
\end{array}\right)
$$


For the metric $g$ and antisymmetric background field $b$ we find

$$
\begin{gathered}
g=\left(\begin{array}{cccccc}
R^{2} & x & 0 & -R^{2} / 2-x & -R^{2} / 2-x & 0 \\
x & R^{2} & x & -R^{2} / 2-x & -R^{2} / 2-x & 0 \\
0 & x & R^{2} & -R^{2} / 2 & -R^{2} / 2 & 0 \\
-R^{2} / 2-x & -R^{2} / 2-x & -R^{2} / 2 & S^{2} & 2 R^{2}-S^{2}+2 x & -u \\
-R^{2} / 2-x & -R^{2} / 2-x & -R^{2} / 2 & 2 R^{2}-S^{2}+2 x & S^{2} & u \\
0 & 0 & 0 & -u & u & V^{2}
\end{array}\right), \\
b=\left(\begin{array}{cccccc}
0 & \alpha & -2 \alpha+2 \beta & \alpha-\beta & \alpha-\beta & 0 \\
-\alpha & 0 & \alpha & -\alpha+\beta & -\alpha+\beta & 0 \\
2 \alpha-2 \beta & -\alpha & 0 & \beta & \beta & 0 \\
-\alpha+\beta & \alpha-\beta & -\beta & 0 & 0 & -\gamma \\
-\alpha+\beta & \alpha-\beta & -\beta & 0 & 0 & \gamma \\
0 & 0 & 0 & \gamma & -\gamma & 0
\end{array}\right)
\end{gathered}
$$

with $R, S, V, x, u, \alpha, \beta, \gamma \in \mathbb{R} . Q^{2}, Q^{4}$ and $Q^{6}$ leave invariant the third complex subspace. This subspace is spanned by the following basis vectors:

$$
w=\left(\begin{array}{c}
0 \\
0 \\
0 \\
n^{1} \\
-n^{1} \\
n^{2}
\end{array}\right), n^{1}, n^{2} \in \mathbb{Z} \quad \text { and } \quad p=\left(\begin{array}{c}
0 \\
0 \\
0 \\
m_{1} \\
-m_{1} \\
m_{2}
\end{array}\right), \quad m_{1}, m_{2} \in \mathbb{Z} .
$$

The $(1,1)$ - and $(2,1)$-modulus belonging to deformations of the third complex coordinates can be defined as

$$
\begin{aligned}
T & =4\left[-\gamma+i \sqrt{-u^{2}+V^{2}\left(S^{2}-R^{2}-x\right)}\right], \\
U & =\frac{1}{2 S^{2}-2 R^{2}-2 x}\left[-u+i \sqrt{-u^{2}+V^{2}\left(S^{2}-R^{2}-x\right)}\right] .
\end{aligned}
$$

Using (2.37) we get for $Z_{\left(1, \Theta^{2}\right)}^{\text {torus }}$ in (2.9) the following expression:

$$
Z_{\left(1, \Theta^{2}\right)}^{\text {torus }}(\tau, \bar{\tau}, T, U)=\sum_{\substack{m_{1}, m_{2} \in \mathbb{Z} \\ n^{1}, n^{2} \in \mathbb{Z}}} e^{2 \pi i \tau\left(2 m_{1} n^{1}+m_{2} n^{2}\right)} e^{\frac{-\pi \tau_{2}}{T_{2} U_{2}}\left|T U n^{2}+T n^{1}-2 U m_{1}+m_{2}\right|^{2}} .
$$

After a Poisson transformation in the momenta (2.39) becomes

$$
\tau_{2} Z_{\left(1, \Theta^{2}\right)}^{\text {torus }}(\tau, \bar{\tau}, T, U)=\frac{1}{2} \sum_{A \in \mathcal{M}} e^{-2 \pi i T \operatorname{det} A} T_{2} e^{\frac{-\pi T_{2}}{\tau_{2} U_{2}}\left|(1, U) A\left(\begin{array}{c}
\tau \\
1
\end{array}\right)\right|^{2}},
$$

where

$$
\mathcal{M}=\left\{\left(\begin{array}{cc}
n_{1} & \frac{1}{2} l_{1} \\
n_{2} & l_{2}
\end{array}\right) \mid n_{1}, n_{2}, l_{1}, l_{2} \in \mathbb{Z}\right\} .
$$

The $N=2$ orbit is $\mathcal{O}=\left\{\left(1, \Theta^{2}\right),\left(\Theta^{2}, 1\right),\left(\Theta^{2}, \Theta^{2}\right),\left(\Theta^{2}, \Theta^{4}\right),\left(1, \Theta^{6}\right),\left(\Theta^{6}, 1\right),\left(\Theta^{6}, \Theta^{6}\right),\left(\Theta^{6}, \Theta^{4}\right)\right.$, $\left.\left(\Theta^{4}, \Theta^{6}\right),\left(\Theta^{6}, \Theta^{2}\right),\left(\Theta^{4}, \Theta^{2}\right),\left(\Theta^{2}, \Theta^{6}\right)\right\} \cup\left\{\left(1, \Theta^{4}\right),\left(\Theta^{4}, 1\right),\left(\Theta^{4}, \Theta^{4}\right)\right\}$. The second set is invariant under $\Gamma$ by itself. Moreover it is a irreducible representation of $\Gamma$. The fundamental orbit consists of $\mathcal{O}_{0}=\left\{\left(1, \Theta^{2}\right),\left(1, \Theta^{4}\right),\left(1, \Theta^{6}\right),\left(\Theta^{4}, \Theta^{2}\right),\left(\Theta^{4}, \Theta^{6}\right)\right\}$. All other sectors can be generated 
from these by acting on $\tau$ with $1, S$ and $S T$. The partition functions for all sectors appearing in $\mathcal{O}_{0}$ agree with the one of $\left(1, \Theta^{2}\right)$. The coefficient $b_{a}^{\left(1, \Theta^{2}\right)}$ of the untwisted sector is two times that of the twisted sectors $\left(\Theta^{2}, 1\right)$ and $\left(\Theta^{2}, \Theta^{2}\right)$. We postpone the further details to the appendix and find the following expression for the threshold corrections (2.5):

$$
\begin{aligned}
\triangle_{a}(T, \bar{T}, U, \bar{U})= & -5 b_{a}^{\left(1, \Theta^{2}\right)} \ln \left[\frac{8 \pi e^{1-\gamma_{E}}}{3 \sqrt{3}} T_{2}|\eta(T)|^{4} U_{2}|\eta(2 U)|^{4}\right] \\
& -5 b_{a}^{\left(1, \Theta^{2}\right)} \ln \left[\frac{8 \pi e^{1-\gamma_{E}}}{3 \sqrt{3}} T_{2}\left|\eta\left(\frac{T}{2}\right)\right|^{4} U_{2}|\eta(U)|^{4}\right] .
\end{aligned}
$$

Again this result is no longer invariant under a symmetry $\Gamma_{T} \times \Gamma_{U}$ but under the group $\Gamma^{0}(2)_{T} \times$ $\Gamma_{0}(2)_{U}$. This symmetry group agrees with the one of the spectrum: The mass formula for the two-dimensional subspace reads

$$
m_{\perp}^{2}=\sum_{\substack{m_{1}, m_{2} \in \mathbb{Z} \\ n^{1}, n^{2} \in \mathbb{Z}}} \frac{1}{T_{2} U_{2}}\left|T U n^{2}+T n^{1}-2 U m_{1}+m_{2}\right|^{2} .
$$

This formula is invariant under the following transformations on $T$ and $U$, respectively:

$$
\begin{gathered}
T \rightarrow T+2 \quad, \quad T \rightarrow \frac{T}{T+1}, \\
U \longrightarrow U+1 \quad, \quad U \rightarrow-\frac{U}{2 U-1} .
\end{gathered}
$$

Together they form the generators of the group $\Gamma^{0}(2)_{T} \times \Gamma_{0}(2)_{U}$. The topological free energy can be calculated to be

$$
\begin{aligned}
F(T, \bar{T}, U, \bar{U}) & =\ln \left[T_{2}|\eta(T)|^{4} U_{2}|\eta(2 U)|^{4}\right]+\ln \left[\frac{T_{2}}{2}\left|\eta\left(\frac{T}{2}\right)\right|^{4} U_{2}|\eta(U)|^{4}\right], \\
\tilde{F}(T, \bar{T}, U, \bar{U}) & =\ln \left[T_{2}\left|\eta\left(\frac{T}{2}\right)\right|^{4} U_{2}|\eta(2 U)|^{4}\right]+\ln \left[T_{2}|\eta(T)|^{4} U_{2}|\eta(U)|^{4}\right],
\end{aligned}
$$

for the two inequivalent lattices $\Lambda_{N}^{\perp}$ and $\left(\Lambda_{N}^{\perp}\right)^{*}$ discussed previously, respectively.

\section{Anomaly cancellation and gauge coupling unification}

In the effective field theory the non-holomorphic part of the threshold corrections calculated before (see (2.20), 2.31) and (2.41)) is reproduced by triangle graphs involving two gauge fields and a connection of the Kähler or sigma-model coordinate transformations [3, 4, 5]. The contribution of these graphs can be easily calculated by considering its supersymmetric completion, the parity odd coupling representing mixed gauge-Kähler or gauge-sigma model coordinate anomalies. In the presence of a Green-Schwarz mechanism, that is, a one-loop coupling of the antisymmetric tensor field to some gauge or auxiliary gauge field, one has to substract its contribution from the total anomaly coefficient. For a generic modulus the one-loop correction to the gauge coupling is then given by

$$
\triangle_{a, F T}=\left(c_{\mathrm{K}}^{a}+c_{\mathrm{C}}^{A, a}-\delta_{\mathrm{GS}}\right) \ln (\operatorname{Im} T) .
$$

Here $c_{\mathrm{K}}^{a}$ and $c_{\mathrm{C}}^{A, a}$ are group-theoretical coefficients characterizing the contribution of the massless spectrum due to the coupling to the Kähler and sigma-model connection, respectively and $\delta_{\mathrm{GS}}$ is 
the coefficient in front of the gauge group independent Green-Schwarz counterterm. For further details we refer the reader to refs. [3, $, 0,5]$. $\delta_{\mathrm{GS}}$ can be determined from the previous string calculation to be

$$
\delta_{\mathrm{GS}}=c_{\mathrm{K}}^{a}+c_{\mathrm{C}}^{A, a}-b_{a}^{N=2} .
$$

Obviously $\triangle_{a, F T}$ is not invariant under modular transformations on the modulus $T$, but only the full expression for the threshold correction including the holomorphic part. Since we have shown in the previous section that the symmetry group of the threshold corrections w.r.t. reparametrizations of the moduli manifold is only a subgroup of the full modular group $\Gamma$, it is clear that the exact expression could not be inferred from the field-theoretical term (3.1) by imposing anomaly cancellation. This is because the relevant subgroup has no longer a single automorphic function as it happened in the case of the group $\Gamma$. Therefore the previous string calculations are necessary to determine the exact form of the full threshold corrections which provide the boundary conditions for the running coupling constants. The renormalization group equation is given by [0, 3,13$]$

$$
\frac{1}{g_{a}^{2}(\mu)}=\frac{k_{a}}{g_{\text {string }}^{2}}+\frac{b_{a}}{16 \pi^{2}} \ln \frac{M_{\text {string }}^{2}}{\mu^{2}}-\frac{1}{16 \pi^{2}} \triangle_{a},
$$

where $k_{a}$ is the Kac-Moody level of the gauge group $a$ and $b_{a}$ is the complete $\beta$-function coefficient of all orbifold sectors. $M_{\text {string }}$ denotes the string scale of the order of the Planck scale. In ref. [7] it was calculated to be $M_{\text {string }}=0.7 g_{\text {string }} \times 10^{18} \mathrm{GeV}$ in the $\overline{\mathrm{DR}}$ scheme. $\triangle_{a}$ is given by (2.20),(2.31) and (2.41) for the $Z_{4}, Z_{6}$ and $Z_{8}$ orbifold models considered in the previous sections, respectively. The unification mass, defined as the scale where the two gauge couplings $g_{a}$ and $g_{b}$ meet can be determined to be

$$
\begin{aligned}
& Z_{4}: \quad M_{X}=M_{\text {string }}\left[\frac{U_{2} T_{2}}{4^{2 / 3}}\left|\eta\left(\frac{T}{2}\right)\right|^{4}|\eta(U)|^{4}\right]^{\frac{b_{b}^{N=2}-b_{a}^{N=2}}{2\left(b_{a}-b_{b}\right)}}, \\
& Z_{6}: \quad M_{X}=M_{\text {string }}\left[\frac{T_{2} U_{2}}{3}\left|\eta(T) \eta\left(\frac{T}{3}\right) \eta(U+2) \eta\left(\frac{U+2}{3}\right)\right|^{2}\right]^{\frac{b_{b}^{N}=2-b_{a}^{N=2}}{2\left(b_{a}-b_{b}\right)}}, \\
& Z_{8}: \quad M_{X}=M_{\text {string }}\left[T_{2} U_{2}\left|\eta(T) \eta\left(\frac{T}{2}\right) \eta(U) \eta(2 U)\right|^{2}\right]^{\frac{b_{b}^{N=2}-b_{a}^{N=2}}{2\left(b_{a}-b_{b}\right)}} .
\end{aligned}
$$

The new functional dependence of the threshold corrections has to be taken into account in phenomenological discussions of the above orbifold models. In refs. 13, 14 numerous orbifolds have been investigated with respect to their predictions for the values of the weak mixing angle $\sin ^{2} \theta_{W}$ and the strong coupling constant $\alpha_{\mathrm{s}}$ assuming the particle content of a minimal supersymmetric standard model. Moreover the various models have been classified using the constraints on the spectrum following from anomaly freedom with respect to duality transformations on the moduli in the planes rotated by all orbifold twists. It is remarkable that although the explicit form of the threshold corrections evaluated above differs from the expressions for the known orbifolds, most of the arguments of ref. [14] go through. Since the symmetry group of the moduli of the unrotated plane is a subgroup of the modular group, the modular weights for the matter fields do not change. Indeed the conditions for the modular weights arising from requiring the correct weak mixing angle and strong coupling constant together with anomaly freedom with respect to the moduli reparametrizations in the completely rotated planes remain valid. What will change 
are the values for the moduli $T$ and $U$ which solve the minimal unification scenario. The fact that $T$ and $U$ are divided by integer numbers in the threshold corrections favours the tendency to higher values and raises the problem of solutions far away from the self-dual point.

\section{Summary and Conclusions}

We derived the threshold corrections to the gauge couplings for the class of toroidal orbifolds models with a generic six-dimensional torus lattice which does not split into a direct sum of orthogonal sublattices. The final expressions (cf. (2.20), (2.31) and (2.41)) do not have the functional form which has been expected by just considering the symmetry of the Kähler potentials: The thresholds are not invariant under the group $\Gamma_{T} \times \Gamma_{U}$ acting on the relevant moduli $T$ and $U$. In contrast they turn out to be functions automorphic only under a subgroup of $\Gamma_{T} \times \Gamma_{U}$. In addition we showed that the spectrum shares this property. This has interesting consequences for the Yukawa couplings between twisted matter fields: the coupling w.r.t. the unrotated plane can be shown to reduce always to a twist-antitwist coupling as a consequence of the point group selection rule [11]. Since its functional dependence on the moduli is the same as that of the spectrum, this special class of Yukawa couplings will have exactly the same symmetry properties as the spectrum and the threshold corrections.

While the non-holomorphic part of the threshold corrections is fixed by the known Kähler potentials up to constant terms, the holomorphic part which describes the massive string states and is necessary for the cancellation of duality anomalies in the effective theory has changed compared to the results of ref. [12, 3]. In contrast formulas where only the weights of modular functions appear, like the transformation behaviour of the vertex operators of the various fields or the superpotential [31], remain untouched by the change of the symmetry group. We want to mention that the symmetry of the spectrum of the completely untwisted sector is not determined by the above considerations. In general a mass formula corresponding to e.g. (2.21) can have quite a complicated dependence on the whole set of moduli fields. This was shown in ref. 32] for the case of the $Z_{7}$ orbifold.

Our above calculations indicate that the significance of the threshold corrections is far from beeing investigated sufficiently. Just a different choice of the lattice already has changed the results in a remarkable way. In more general - and more realistic - models, like orbifolds with Wilson lines [33] or vacuum exspectation values of certain scalar fields, there will arise additional contributions to the thresholds of an up to now unknown size. This fact might change the phenomenological prospects of such models.

\section{Acknowledgements}

We would like to thank Lance Dixon, Albrecht Klemm and especially Hans Peter Nilles for helpful discussions.

\section{Appendix}

In this appendix we present the technical details necessary for evaluating the integral and infinite sum in the previous formulas. 


\section{$Z_{4}$ orbifold}

Under a $\Gamma_{0}(2)$ transformation $\tau \rightarrow \frac{a \tau+b}{c \tau+d}$ (with $a d-b c=1, c=0 \bmod 2$ ), eq. (2.17) remains invariant if we transform the four integral numbers $n_{1}, n_{2}, l_{1}$ and $l_{2}$ in the following way:

$$
\left(\begin{array}{cc}
n_{1}^{\prime} & n_{2}^{\prime} \\
l_{1}^{\prime} & l_{2}^{\prime}
\end{array}\right)=\left(\begin{array}{cc}
a & c / 2 \\
2 b & d
\end{array}\right)\left(\begin{array}{cc}
n_{1} & n_{2} \\
l_{1} & l_{2}
\end{array}\right) .
$$

Clearly $(2.17)$ is only invariant under the group $\Gamma_{0}(2)$ acting on $\tau$. To proceed similar as in the appendix of ref. [12] we have to divide the set of all integral two by two matrices into equivalence classes under the group $\Gamma_{0}(2)$. There are three kinds of orbits:

1. The zero matrix whose contribution to (2.17) and the integral (2.5) is denoted by $I_{1}$. The integration over $\tilde{\mathcal{F}}=\{1, S, S T\} \mathcal{F}$ yields

$$
I_{1}=\frac{3}{2} \times \frac{\pi}{3} \frac{T_{2}}{2}
$$

2. All matrices with non-zero determinants. Since the fundamental region of $\Gamma_{0}(2)$ is $\tilde{\mathcal{F}}=$ $\{1, S, S T\} \mathcal{F}$ we obtain the following representatives:

$$
\left(\begin{array}{ll}
k & j \\
0 & p
\end{array}\right),\left(\begin{array}{cc}
0 & -p \\
k & j
\end{array}\right),\left(\begin{array}{cc}
0 & -p \\
k & j+p
\end{array}\right), 0 \leq j<k, \quad p \neq 0
$$

One can generate all $G L(2, \mathbb{Z})$ matrices from these three matrices by acting ${ }^{3}$ on them with $\Gamma_{0}(2)$. Their contribution to (2.5) together with that of $I_{1}$ is

$$
I_{1}+I_{2}=-\frac{3}{2} \times 4 \operatorname{Re} \ln \eta\left(\frac{T}{2}\right) .
$$

3. The orbits of matrices with zero determinant can be obtained from the two representatives

$$
\left(\begin{array}{ll}
0 & 0 \\
j & p
\end{array}\right),\left(\begin{array}{ll}
j & p \\
0 & 0
\end{array}\right), j, p \in \mathbb{Z},(j, p) \neq(0,0) .
$$

Note that not all $\Gamma_{0}(2)$ group elements acting on (3) lead to different matrices. A $T^{n}$ transformation on $\tau$ does not modify the first of the above matrices because this change in $\tau$ is accompanied by a multiplication with $\left(\begin{array}{cc}1 & 0 \\ 2 n & 1\end{array}\right)$ on the matrix. Similar a $S T^{2 n} S$ transformation on $\tau$ does not change the second representative. Following [12] the contribution $I_{3}$ of the third class together with the last term of (2.5) can be calculated to be

$$
\begin{aligned}
I_{3}= & -4 \operatorname{Re} \ln \eta(U)-\ln \left(\frac{T_{2}}{4} U_{2}\right)+\left(\gamma_{E}-1-\ln \frac{8 \pi}{3 \sqrt{3}}\right) \\
& -\frac{1}{2} \times 4 \operatorname{Re} \ln \eta(U)-\frac{1}{2} \times \ln \left(T_{2} U_{2}\right)+\frac{1}{2} \times\left(\gamma_{E}-1-\ln \frac{8 \pi}{3 \sqrt{3}}\right) .
\end{aligned}
$$

The first matrix in (3) has to be integrated over the half-band $\left\{\tau \in \mathbb{C}\left|\tau_{2}>0,\right| \tau_{1} \mid<\frac{1}{2}\right\}$ as explained in ref. [12]. In contrast the second matrix has to be integrated over a half-band with the double width in $\tau_{1}$.

\footnotetext{
${ }^{3}$ To get also the matrices with an overall minus sign one has to make the change $(a, b, c, d) \rightarrow(-a,-b,-c,-d)$ which gives an additional factor of two (cf. 12]).
} 


\section{$Z_{6}$ orbifold}

Acting with $\Gamma_{0}(3)$ on $\tau$ leaves invariant eq. (2.29) if the momentum and winding numbers are transformed unimodularly as

$$
\begin{aligned}
& n_{1}^{\prime}=a n_{1}+c l_{1}, \quad n_{2}^{\prime}=a n_{2}+\frac{c}{3} l_{2}, \\
& l_{1}^{\prime}=b n_{1}+d l_{1}, \quad l_{2}^{\prime}=3 b n_{2}+d l_{2} .
\end{aligned}
$$

Again these transformations can be written in the form of (1) restricting to $l_{1} \in 3 \mathbb{Z}$. Moreover the exponential in (2.29) has to be replaced by

$$
e^{-2 \pi i T\left(n_{1} \frac{l_{2}}{3}-n_{2} \frac{l_{1}}{3}\right)} e^{\frac{-\pi T_{2}}{\tau_{2} U_{2}^{\prime}}\left|n_{1} \tau+\frac{l_{1}}{3}+U^{\prime} n_{2} \tau+U^{\prime} \frac{l_{2}}{3}\right|^{2}}, l_{1} \in 3 \mathbb{Z} .
$$

For $\Gamma_{0}(3)$ there is a fourth orbit additional to those of (2). It is generated by the matrix

$$
\left(\begin{array}{cc}
0 & -p \\
k & j+2 p
\end{array}\right) \quad, \quad 0 \leq j<k \quad, \quad p \neq 0 .
$$

The four matrices with non-zero determinants together with the zero matrix give rise to the contribution

$$
I_{1}+I_{2}=-4 \operatorname{Re} \ln \eta\left(\frac{T}{3}\right)-4 \operatorname{Re} \ln \eta(T)
$$

Since the second matrix of (3) remains invariant under a $S T^{3 n} S$-transformation on $\tau$, the contribution of this matrix in (2.5) has to be integrated over three neighbouring half-bands. In this way one obtains for the matrices with zero-determinants the following result:

$$
\begin{aligned}
I_{3}= & -4 \operatorname{Re} \ln \eta\left(\frac{U^{\prime}}{3}\right)-\ln \left(\frac{T_{2}}{3} \frac{U_{2}}{3}\right)+\left(\gamma_{E}-1-\ln \frac{8 \pi}{3 \sqrt{3}}\right) \\
& -4 \operatorname{Re} \ln \eta\left(U^{\prime}\right)-\ln \left(T_{2} U_{2}\right)+\left(\gamma_{E}-1-\ln \frac{8 \pi}{3 \sqrt{3}}\right) .
\end{aligned}
$$

\section{$Z_{8}$ orbifold}

A $\Gamma_{0}(2)$ transformation on $\tau$ is equivalent to the following unimodular redefinition of the integral numbers in (2.40):

$$
\begin{aligned}
& n_{1}^{\prime}=a n_{1}+\frac{c}{2} l_{1}, \quad n_{2}^{\prime}=a n_{2}+c l_{2}, \\
& l_{1}^{\prime}=2 b n_{1}+d l_{1}, \quad l_{2}^{\prime}=b n_{2}+d l_{2} .
\end{aligned}
$$

Again this expression can be written similar as in (11) if one restricts $l_{2}$ to even numbers. The exponentials of (2.40) have to be modified to

$$
e^{-2 \pi i T\left(n_{1} \frac{l_{2}}{2}-n_{2} \frac{l_{1}}{2}\right)} e^{\frac{-\pi T_{2}}{\tau_{2} U_{2}}\left|n_{1} \tau+\frac{l_{1}}{2}+U n_{2} \tau+U \frac{l_{2}}{2}\right|^{2}}, l_{2} \in 2 \mathbb{Z} .
$$

For the orbits we can take (2) and (3) but with even $l_{2}$. Their contribution together with that of the zero matrix can be calculated to be

$$
I_{1}+I_{2}=-2 \times 4 \operatorname{Re} \ln \eta\left(\frac{T}{2}\right)-\frac{1}{2} \times 4 \operatorname{Re} \ln \eta(T) .
$$


The integral from the two orbits with zero determinant is:

$$
\begin{aligned}
I_{3}= & -4 \operatorname{Re} \ln \eta(2 U)-\ln \left(T_{2} U_{2}\right)+\left(\gamma_{E}-1-\ln \frac{8 \pi}{3 \sqrt{3}}\right) \\
& -4 \operatorname{Re} \ln \eta(U)-\ln \left(T_{2} U_{2}\right)+\left(\gamma_{E}-1-\ln \frac{8 \pi}{3 \sqrt{3}}\right) .
\end{aligned}
$$

\section{References}

[1] P. Candelas, G. Horowitz, A. Strominger and E. Witten, Nucl. Phys. B 258 (1985) 46

[2] L. Dixon, J. Harvey, C. Vafa and E. Witten, Nucl. Phys. B 261 (1985) 678; B 274 (1986) 285;

L. E. Ibáñez, J. Mas, H. P. Nilles and F. Quevedo, Nucl. Phys. B 301 (1988) 157

[3] J. P. Derendinger, S. Ferrara, C. Kounnas and F. Zwirner, Nucl. Phys. B 372 (1992) 145

[4] J. Louis, Non-harmonic Gauge Couplings Constants in Supersymmetry and Superstring Theories, Proceedings Second International Symposium on Particles, Strings and Cosmology, World Scientific 1991

[5] G. Cardoso and B. Ovrut, Sigma Model Anomalies in Supergravity and String Theory, Philadelphia preprint UPR-0520T; Coordinate and Kähler Sigma-Model Anomalies and their Cancellation in String Effective Field Theories, UPR-0502T

[6] L. Dixon, V. Kaplunovsky and J. Louis Nucl. Phys. B 329 (1990) 27

[7] V. Kaplunovsky, Nucl. Phys. B 307 (1988) 145 and Erratum, Stanford preprint ITP-838/1992

[8] I. Antoniadis and T. R. Taylor, String Loop Corrections to Gauge and Yukawa Couplings, Palaiseau preprint, CPTH-C218.0193

[9] I. Antoniadis, E. Gava and K. S. Narain, Nucl. Phys. B 383 (1992) 93

[10] M. Cvetič, J. Louis and B. Ovrut Phys. Lett. B 206 (1988) 227

[11] L. Dixon, D. Friedan, E. Martinec and S. Shenker, Nucl. Phys. B 282 (1987) 13;

D. Jungnickel, J. Lauer, M. Spaliński and S. Stieberger, Mod. Phys. Lett. A 33 (1992) 3059;

J. Erler, D. Jungnickel, M. Spaliński and S. Stieberger, Higher Twisted Sector Couplings of $Z_{N}$ Orbifolds, Munich preprint MPI-Ph/92-56, to appear in Nucl. Phys. B;

S. Stieberger, Phys. Lett. B 300 (1993) 347

[12] L. Dixon, V. Kaplunovsky and J. Louis Nucl. Phys. B 355 (1991) 649

[13] L. E. Ibáñez, D. Lüst and G. G. Ross, Phys. Lett. B 272 (1991) 251

[14] L. E. Ibáñez and D. Lüst, Nucl. Phys. B 382 (1992) 305

[15] J. P. Derendinger, L. E. Ibáñez and H. P. Nilles Phys. Lett. B 155 (1985) 65;

M. Dine, R. Rohm, N. Seiberg and E. Witten, Phys. Lett. B 156 (1985) 55;

H. P. Nilles and N. Olechowski, Phys. Lett. B 248 (1990) 268;

A. Font, L. E. Ibáñez, D. Lüst and F. Quevedo, Phys. Lett. B 245 (1990) 401;

S. Ferrara, N. Magnoli, T.R. Taylor and G. Veneziano, Phys. Lett. B 245 (1990) 409

[16] L. E. Ibáñez and H. P. Nilles, Phys. Lett. B 169 (1986) 354;

H. P. Nilles, Phys. Lett. B 180 (1986) 240

[17] M. Cvetič, A. Font, L. E. Ibáñez, D. Lüst and F. Quevedo, Nucl. Phys. B 361 (1991) 194

[18] H. Ooguri and C. Vafa, Nucl. Phys. B 361 (1991) 469 
[19] S. Ferrara, C. Kounnas, D. Lüst and F. Zwirner, Nucl. Phys. B 365 (1991) 431

[20] J. Erler and A. Klemm, Comment on the Generation Number in Orbifold Compactifications, Munich preprint MPI-Ph/92-60, to appear in Comm. Math. Phys.

[21] T. Apostol, Modular Functions and Dirichlet Series in Number Theory, 2nd Edition 1990, GTM 41, Springer-Verlag

[22] G. Moore, Nucl. Phys. B 293 (1987) 139

[23] I. Antoniadis, J. Ellis, R. Lacaze and D. V. Nanopoulos, Phys. Lett. B 268 (1991) 188

[24] T. Banks and L. Dixon, Nucl. Phys. B 307 (1988) 93

[25] J. Lehner, Lectures on Modular Forms, National Bureau of Standards, Applied Mathematics Series 61

[26] M. Spaliński, Nucl. Phys. B 377 (1992) 339

[27] M. Spaliński, Phys. Lett. B 275 (1992) 47;

J. Erler, D. Jungnickel and H. P. Nilles, Phys. Lett. B 276 (1992) 303

[28] K. S. Narain, M. H. Sarmadi and E. Witten, Nucl. Phys. B 279 (1987) 369

[29] R. Dijkgraaf, E. Verlinde and H. Verlinde, On Moduli Spaces of Conformal Field Theories with $c \geq 1$, Proceedings Copenhagen Conference, Perspectives in String Theory, World Scientific, Singapore, 1988

[30] J. Lauer, J. Mas and H. P. Nilles, Nucl. Phys. B 351 (1991) 353

[31] S. Ferrara, D. Lüst, A. Shapere and S. Theisen, Phys. Lett. B 225 (1989) 363;

S. Ferrara, D. Lüst and S. Theisen, Phys. Lett. B 233 (1989) 147

[32] J. Erler and M. Spaliński, Modular Groups for Twisted Narain Models, Munich preprint MPI-Ph/9261

[33] L. E. Ibáñez, H. P. Nilles and F. Quevedo, Phys. Lett. B 187 (1987) 25;

L. E. Ibáñez, H. P. Nilles and F. Quevedo, Phys. Lett. B 192 (1987) 333;

L. E. Ibáñez, J. E. Kim, H. P. Nilles and F. Quevedo, Phys. Lett. B 191 B (1987) 3. 